\title{
A Ray-Tracing Method Based on the Triangular Grid Approach and Application to Propagation Prediction in Urban Environments
}

\author{
Zhengqing Yun, Member, IEEE, Zhijun Zhang, Member, IEEE, and Magdy F. Iskander, Fellow, IEEE
}

\begin{abstract}
This paper presents a fast ray-tracing procedure based on triangular division of the propagation environments. Unlike other accelerating methods that are mostly based on pre-establishment of visibility, this method does not require knowledge of the position information of the base station and/or receiving antennas and is thus more general. Although the triangulation is done in a two-dimensional (2-D) plane, this method is suitable for three-dimensional (3-D) simulations when a proper data structure for buildings is constructed. Validation results show good agreement between calculated and measured data from the European COST 231 project. The improvement in the computational efficiency is clearly demonstrated in examples.
\end{abstract}

Index Terms-Mobile communication, prediction methods, ray tracing, urban propagation.

\section{INTRODUCTION}

$\mathbf{R}$ ADIO wave propagation models are necessary for the implementation of a mobile radio system. With the rapid growth of wireless communications, the cell sizes are getting smaller and site-specific propagation information is needed for the design of mobile systems. During the past decade, there were many proposed theoretical and measurement-based propagation models for microcells in urban environments. At first, some empirical or theoretical models (formulas) were developed as extensions of the models suitable for macrocells. Factors such as the heights of antennas and buildings, density of buildings, line-of-sight (LOS) or non-LOS propagations, geometry of the street grid, etc., are included [1]-[8]. A good review of these models can be found in [9]. Later on, propagation mechanisms were extensively investigated and the ray theory emerged as a highly promising procedure for providing accurate, site-specific means to obtain useful simulation results [10]-[12]. It should be noted that ray tracing can also serve as a starting point for statistical modeling of wireless channels [13]-[15]. According to the ray optics and the uniform theory of diffraction (UTD), propagation mechanisms may include direct LOS, reflected, transmitted, diffracted, scattered rays, and some combined rays, which complicates and in many realistic propagation environments, slows down the calculation procedure.

Manuscript received June 28, 2001; revised December 19, 2001.

Z. Yun, and Z. Zhang are with The Hawaii Center for Advanced Communications, College of Engineering, University of Hawaii at Manoa, HI 96822 USA.

M. F. Iskander was with the Department of Electrical Engineering, University of Utah, Salt Lake City, Utah. He is now with The Hawaii Center for Advanced Communications, College of Engineering, University of Hawaii at Manoa, HI 96822 USA.

Publisher Item Identifier S 0018-926X(02)05457-1.
It was also found that simulating a realistic 3-D propagation environment using the standard shooting-and-bouncing ray (SBR) method presents a considerable challenge due to the geometric and physical complexity of the environments. It leads to the need for huge and often unavailable computation resources. To overcome this difficulty, simplified models were proposed to only include the contributions from main rays and/or to only consider the main geometric and physical characteristics of buildings and terrains. For example, walls with complex structures are approximated by uniform walls and the tops of buildings are assumed flat, etc. Also, in an urban environment, transmitted rays through buildings are assumed small and are hence neglected. Simplified ray-tracing models are useful, however, in providing reasonably accurate results for some propagation environments including, for example, the case when the base-station antenna is well below the average height of the buildings [16], [17]. In this case, the over-rooftop propagation is negligible and the lateral rays-including LOS, reflected, and diffracted rays-are the most important ones that need to be taken into account. Other approximations that may be used to improve accuracy include the calculation of the rooftop-diffracted rays as well as diffracted-reflected rays in the propagation model [1], [4], [18]-[21].

A more recent advanced simplified model is the vertical plane launch (VPL) model [22], [23]. The VPL technique employs the standard SBR method in the horizontal plane while using a deterministic approach to find the vertical displacement of the unfolded ray paths. This method is valid when the walls are vertical, and similar methods can be found in [24], [25]. Full 3-D ray-tracing methods have also been developed [26] and comparisons between different methods and their applicability can be found in [27]-[30].

Another approach in improving computational efficiency and accuracy of the ray-tracing-based methods is the application and development of geometric algorithms. Basically, the overhead of computation in the ray-tracing algorithm is related to the determination of the intersection of a ray and an object (a wall surface, a ground plane, or a wall edge, etc.) in the propagation environments. This geometric testing can consume more than $90 \%$ of CPU time for a naïve SBR algorithm [31]. Several techniques have been proposed to reduce the time spent on geometric testing using preprocessing of the propagation environments. Some methods in computational geometry and computer graphics, e.g., the bounding box method, can be used to reduce the number of intersection tests. Other methods using 


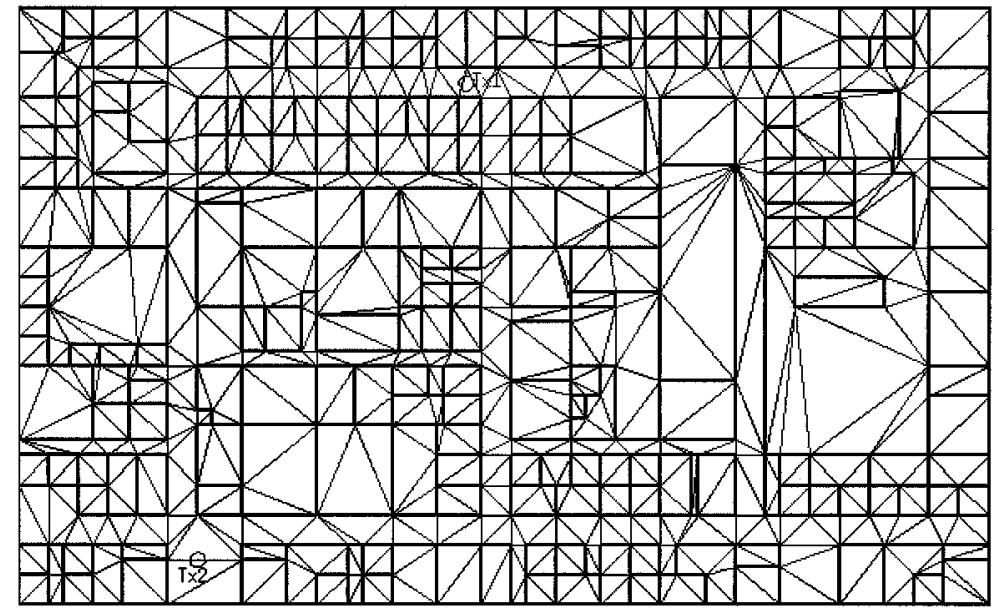

Fig. 1. Triangulation of the third floor of an office building and positions of two base stations.

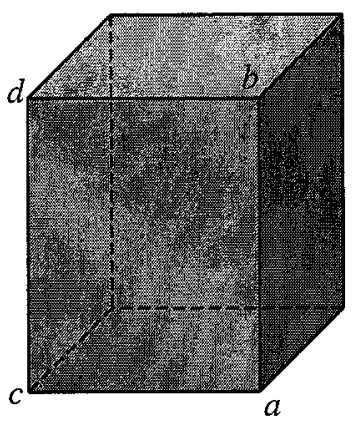

(a)

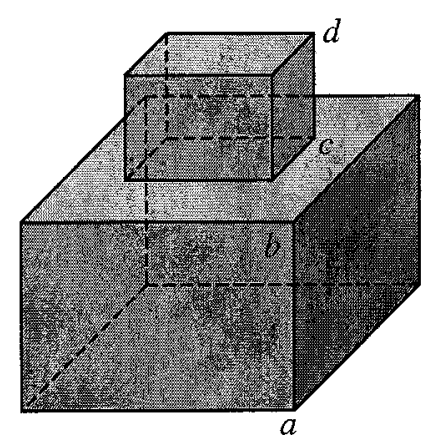

(b)

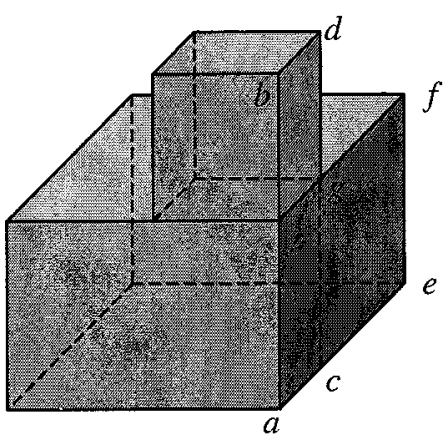

(c)

Fig. 2. Building models. (a) Simple building and (b) (c) stacked buildings.

visibility between transmit antenna (Tx)/receive antenna ( $\mathrm{Rx})$ and wall surfaces (edges) can reduce the number of candidate geometric objects to be tested [16], [26], [32]. These visibilitybased methods are usually Tx-and/or Rx-specific and the preprocessing procedure needs to be repeated for each $\mathrm{Tx}$ and/or Rx position.

A fast ray-traversing algorithm developed in [33], based on uniform rectangular space division, is modified and applied to some environments, and higher efficiency is achieved over the visibility method [34]. Two-dimensional (2-D) results show that, on average, the computation time of the new method is $15 \%$ of that of the visibility method.

This paper is an elaboration and extension of the ideas presented in [35] for 2-D cases. Earlier calculations were extended to deal with 3-D urban environments while using the 2-D triangular grid method as described earlier. The new method is not $\mathrm{Tx} / \mathrm{Rx}$ specific and can serve as a basis for many different ray-tracing approaches, e.g., VPL and over-rooftop models. The obtained 3-D simulation results were compared with experimental data from the European COST 231 project for Munich City in Germany [9]. It should be noted that a similar triangular grid method was briefly described in [36] for indoor environments.

\section{TRIANGUlar GRID-BASED RAY-TRACING APPROACH}

The ray-tracing engine used in this paper is based on the 2-D ray-tracing algorithm proposed in [35]. The basic solution procedure was described earlier and simplified examples were used to illustrate its advantages and usefulness. In this paper, we will start by illustrating its computational efficiency advantage in a realistic propagation environment. A numerical experiment is carried out to compare results from the proposed method with those of the visibility method in an indoor office building as shown in Fig. 1. Two positions of base station antennas were selected, Tx1 and Tx2, as shown in Fig. 1. It may be seen from the figure that the numbers of edges visible to Tx1 and Tx2 are different and the numbers of edges visible to each edge in this case are also different.

To mimic an urban environment, only reflection rays are traced. For each Tx position, the visible edges are determined in the preprocess. The visible edges to each edge in the entire region are also predetermined. Three hundred and sixty rays are launched uniformly in all directions for each Tx and each ray is traced to 40 reflections. This large reflection number is selected to help include as many edges as possible with different numbers of visible edges. No reception test is included. 


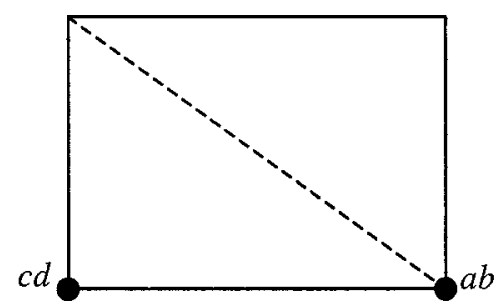

(a)

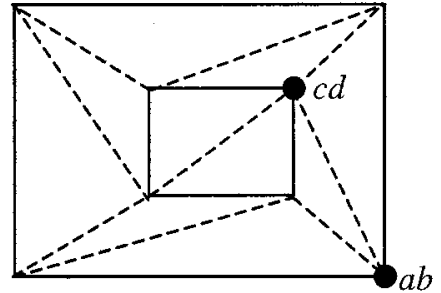

(b)

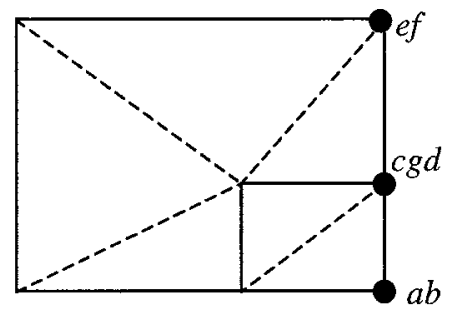

(c)

Fig. 3. 2-D representation of buildings in Fig. 2 and typical triangulations. (a) Simple building and (b) (c) stacked buildings.

The simulation results show that for Tx1, the CPU time ratio of this method over the visibility method is about $25 \%$, while for $\mathrm{Tx} 2$, the ratio is $30 \%$. These calculations, hence, show significant computational advantage of the proposed method.

\section{2-D TRIANGULATION OF REALISTIC PROPAGATION ENVIRONMENTS}

\section{A. 3-D Building Modeling}

In the following calculations, it is assumed that all the buildings are stacked prisms with polygon cross-sections as shown in Fig. 2. These building geometries are projected onto a horizontal plane, i.e., $x$ - $y$ plane, as shown in Fig. 3. The projection of a vertical wall can be represented by two neighboring vertices in the 2-D plane. For example, wall $a b d c$ in Fig. 2(a) may be represented by vertices $a b$ and $c d$. A vertex is actually a vertical edge and has two heights associated with it. For example, vertex $a b$ contains the heights of vertices $a$ and $b$. For simplicity, the height of $a$ is called the ground height, while the height of $b$ is called the vertex height. It is assumed that the two vertex heights of a wall are identical. This means the rooftops of buildings are level-flat polygons. Special attention should be paid to the situation in Fig. 2(c). The walls $a b d c$ and $c e$ f $g$ have a common vertex cgd in the 2-D plane. This suggests that in some cases we may need three parameters to represent the height information correctly, i.e., the heights of $c, d$ and $g$. Fortunately, we can still use two parameters to deal with this situation. The first parameter is the ground height (height of $c$ ). The second is the larger value of the heights of vertices $d$ and $g$. When the height information is needed for a wall, the vertex heights of the two vertices defining the wall are compared and the wall height is always equal to the smaller value of these two heights. For example, when we have to determine the height of wall abdc in Fig. 2(c), we have two vertex heights of $b$ and $d$. Since they have equal values, the wall height is equal to either the height of $b$ or $d$. When we need the height of wall $c g f e$, we have two vertex heights of $d$ and $f$. Since the height of $f$ is less than the height of $d$, the wall height is considered equal to the height of $f$.

\section{B. Triangulation}

When all the buildings in a propagation environment are modeled and represented as described in the previous section (if there are buildings which cannot be presented in this way, approximation will be used), standard triangulation methods can be employed to triangulate the propagation region. (Commercial software or free codes on the Internet can be employed for the fast triangulation.) This paper uses the constrained triangulation of a planar straight-line graph (PSLG) so that a minimum number of dummy edges will be added. An outer boundary, which is usually a polygon, is also added to enclose the environment. A program developed by J. R. Shewchuk, triangle.c [37], is used for the triangulation of the resulting 2-D representation of the propagation domain.

\section{2- AND 3-D LATERAL RAY-TRACING ALGORITHMS}

The basic ray-tracing algorithm for the 2-D case can be found in [35]. Here we give some more details regarding the data structure of the triangular grid and its extended use in modeling 3-D environments.

\section{A. Data Structure of the Triangular Grid}

After triangulation, the topological information is processed and stored into an array. The main entry of the array is a triangle. From a triangle, one can determine its vertices and the three neighboring triangles. The information of wall material for each edge in a triangle is also stored. In particular, the dummy edge (the nonwall edge) is characterized by a wall material index -1 . Edge direction in a triangle is also included for the purpose of fast ray tracing.

\section{B. Data Structure of the Ray Associated With the Triangular Grid}

A ray can be represented by two vectors, one is the position vector $\boldsymbol{p}$, and the other is the direction vector $\boldsymbol{d}$, as shown in Fig. 4. A series of these vector pairs can represent a ray originating from a source point, reflected (or transmitted, diffracted) several times and then arriving at the field (observation) point.

For fast ray tracing based on the triangular grid, it is natural to associate a ray with an edge (for reflected and transmitted rays) or a vertex (for diffracted rays) in a triangle. Fig. 5 shows how a ray is related to an edge. For example, the ray in Fig. 5 is first related to edge $E_{1}$ and triangle $T_{2}$, denoted $\left(E_{1}, T_{2}\right)$, then as it travels through the dummy edge $e_{2}$ and enters triangle $T_{3}$, it is related to $\left(e_{2}, T_{3}\right)$, etc. This process may be represented in general terms as

$$
\begin{aligned}
\left(E_{1}, T_{2}\right) & \rightarrow\left(e_{2}, T_{3}\right) \rightarrow\left(E_{3}, T_{3}\right) \rightarrow\left(e_{2}, T_{2}\right) \rightarrow\left(e_{4}, T_{7}\right) \\
& \rightarrow\left(E_{\tilde{5}}, T_{7}\right) \ldots
\end{aligned}
$$




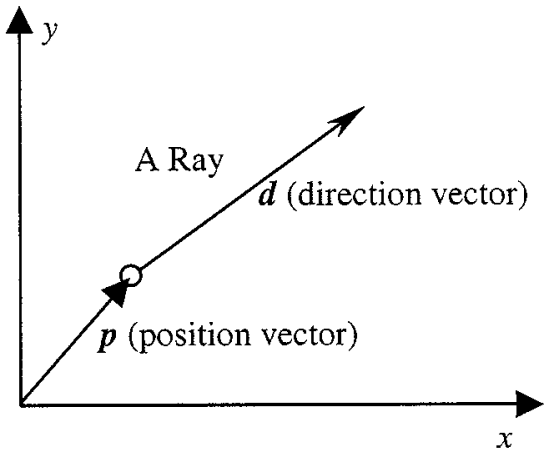

Fig. 4. Definition of a generic ray, located at $\boldsymbol{p}$ and pointed in direction $\boldsymbol{d}$.

This provides a natural data structure for a ray, in terms of class when using $\mathrm{C}++$

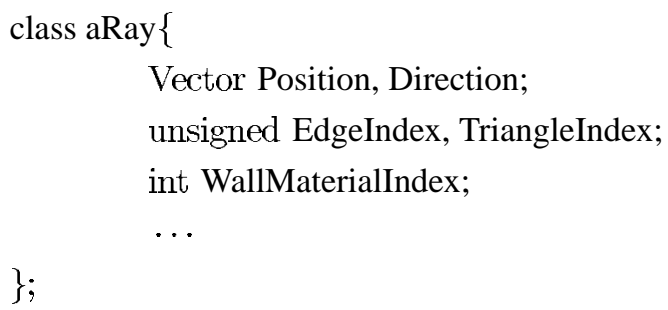

where WallMaterialIndex is used to characterize the wall material. It is set to -1 for the dummy edge and -2 for the outer boundary. Other information can be added to the aRay class in the realistic implementation. A list of class arrays can then represent a ray originating from a source point, reflected (or transmitted, diffracted) several times, and then arriving at a field point.

\section{Initialization of a Ray}

When a source point (antenna) Tx in position $p_{0}$ is given, we first determine the triangle, $T_{0}$, containing it (Tx may be on an edge of a triangle). This is a typical planar point location problem and can be efficiently solved by standard algorithm, e.g., monotone subdivisions [38]. It should be noted that the triangular grid is itself a monotone subdivision and can thus be straightforwardly solved. Then a ray is launched from $\boldsymbol{p}_{0}$ in direction $\boldsymbol{d}_{0}$, see Fig. 6 . This first ray segment is usually not related to any edge and is denoted $\left(-1, T_{0}\right)$.

To trace the ray, we have to determine the first edge that this ray will hit. This can be done by looking at the signs of two cross products of 2-D vectors. First, connect $p_{0}$ to $V_{0}$, the vertex opposite the edge $e_{0}$ in triangle $T_{0}$, then obtain a vector $\boldsymbol{p}_{0} \boldsymbol{V}_{0}$. Take the cross product of $\boldsymbol{d}_{0}$ (original direction) and $\boldsymbol{p}_{0} \boldsymbol{V}_{0}$, i.e., $a=\boldsymbol{d}_{0} \times \boldsymbol{p}_{0} \boldsymbol{V}_{0}$. If $a>0$, the ray will not hit the edge $e_{1}$. If $a<0$, the ray will not hit $e_{2}$. Then, for the case $a<0$ in Fig. 6 , connect $\boldsymbol{p}_{0}$ to $\boldsymbol{V}_{1}$ and take the cross product, $b=\boldsymbol{d}_{0} \times \boldsymbol{p}_{0} \boldsymbol{V}_{1}$. If $b>0$, the ray will hit $e_{1}$; if $b<0$, the ray will hit $e_{0}$. If $a=0$ or $b=0$, a vertex will be hit, which means a vertical wall edge is hit and diffraction will take place. Now we have a ray trajectory

$$
\left(-1, T_{0}\right) \rightarrow\left(e_{1}, T_{1}\right) \rightarrow \cdots,
$$

and the initialization of a ray is achieved.

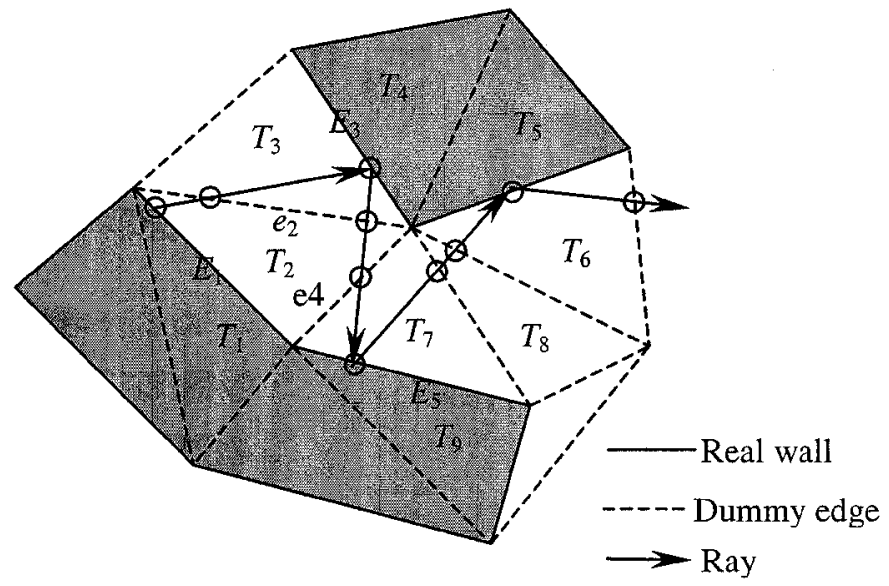

Fig. 5. Relationship between rays and edges in triangles.

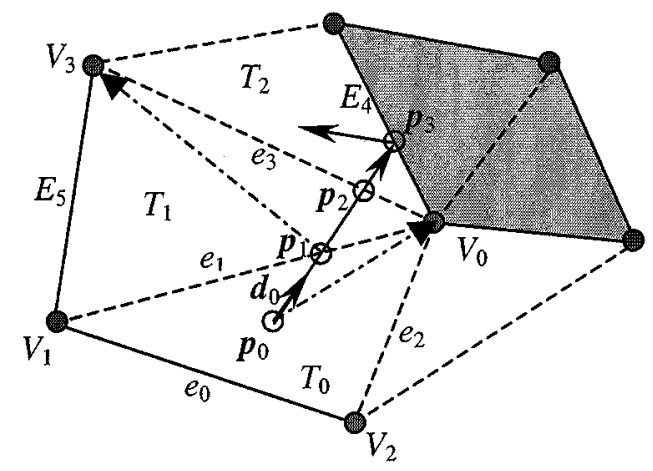

Fig. 6. Initialization of a ray and the determination of the first edge the ray will hit.

\section{2-D Ray-Tracing Procedure}

The remaining part of the proposed ray-tracing procedure is a recursive approach. In Fig. 6, when we trace the ray further, i.e., to find the next edge to be hit, we only have to do one cross-product test. For example, to determine the intersection of the ray with the appropriate edge, connect $\boldsymbol{p}_{1}$ to the opposite vertex of $e_{1}, V_{3}$, in triangle $T_{1}$ and calculate $c=\boldsymbol{d}_{1} \times \boldsymbol{p}_{1} V_{3}$. Here $\boldsymbol{d}_{1}$ is the direction vector of ray $\left(e_{1}, T_{1}\right)$ and is equal to $\boldsymbol{d}_{0}$ since $e_{1}$ is a nonwall edge that did not cause reflection or diffraction of the incident ray. If $c>0$, then edge $e_{3}$ will be hit; if $c<0$, edge $E_{4}$ is hit; if $c=0$, vertex $V_{3}$ is hit.

This test is equivalent to the test of calculating $c=\boldsymbol{d}_{0} \times$ $p_{0} V_{3}$ since no reflection or diffraction occurs. This leads to the fact that there is no need to calculate the position vector on a dummy edge. It is especially useful when a ray is traversing a large number of dummy edges and this will result in a significant saving in CPU time.

\section{E. Reception Test and Diffraction}

A ray is received by a receiving antenna when it hits the reception sphere (or circle, for 2-D cases). To determine if diffraction occurs, the two end points of the wall edge will serve as receiving points and will be tested if the ray hits the corresponding reception sphere. If yes, diffraction occurs. 


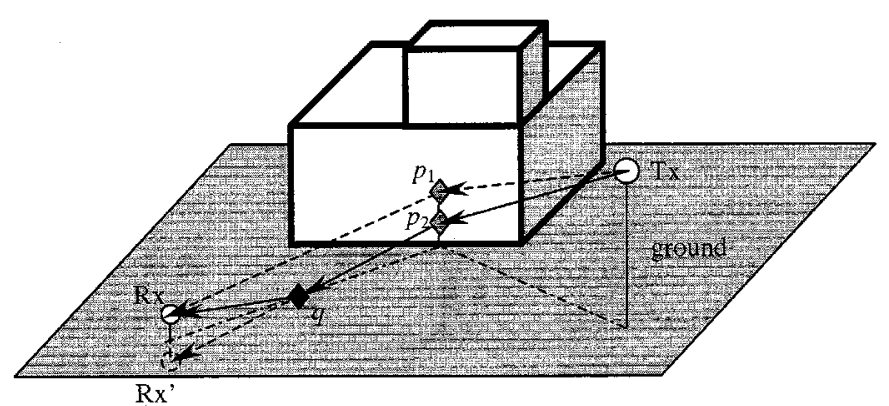

(a)

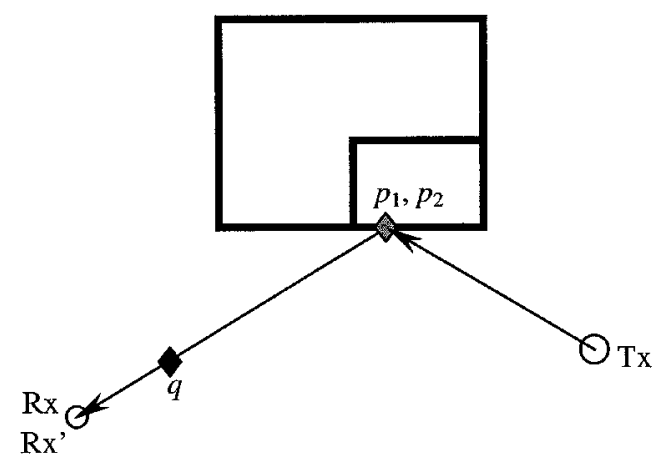

(b)

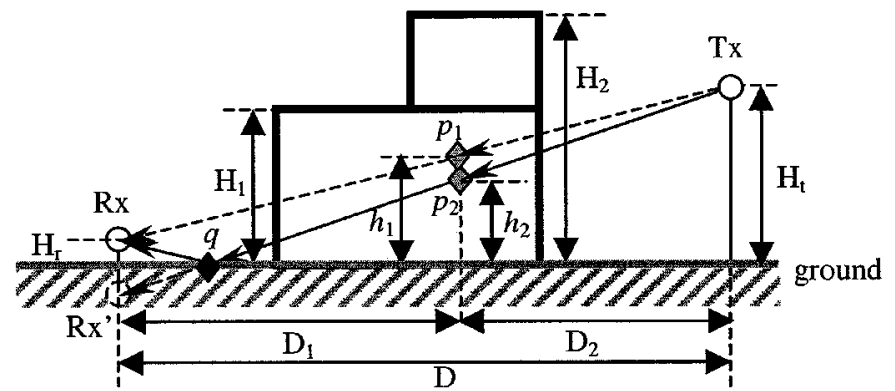

(c)

Fig. 7. Geometries of wall-and wall-ground-reflected rays. Rx' is teh image of Rx with respect to the ground plane. Tx- $p_{1}-\mathrm{Rx}$ is the wall-reflected ray and $\mathrm{Tx}-p_{2}-q-\mathrm{Rx}$ is the wall-ground-reflected ray. (a) Perspective view of rays. (b) Top (projected) view of rays. (c) Unfolded rays.

\section{F. 3-D Lateral Ray Tracing Based on the 2-D Triangle Grid}

For the 3-D ray tracing, this paper proposes a simple method based on the 2-D method. Without loss of generality, we consider the lateral reflected ray tracing. Suppose we trace a 2-D ray and the ray is received by a receiving antenna. In this case, we have to determine the corresponding 3-D trajectory of this ray. The 3-D ray may not be a real ray because the heights of walls associated with it play a significant role. It is easy to calculate the hit point height on a vertical wall using the information of the 2-D ray. If the calculated height is larger than the height of the wall, the ray will not be reflected by the wall and no further action is needed. If the ray is valid (i.e., intercepted by the wall), the received field or power associated with the ray will be calculated. A 2-D ray may represent a 3-D ray which undergoes a ground reflection. This is due to the fact that the

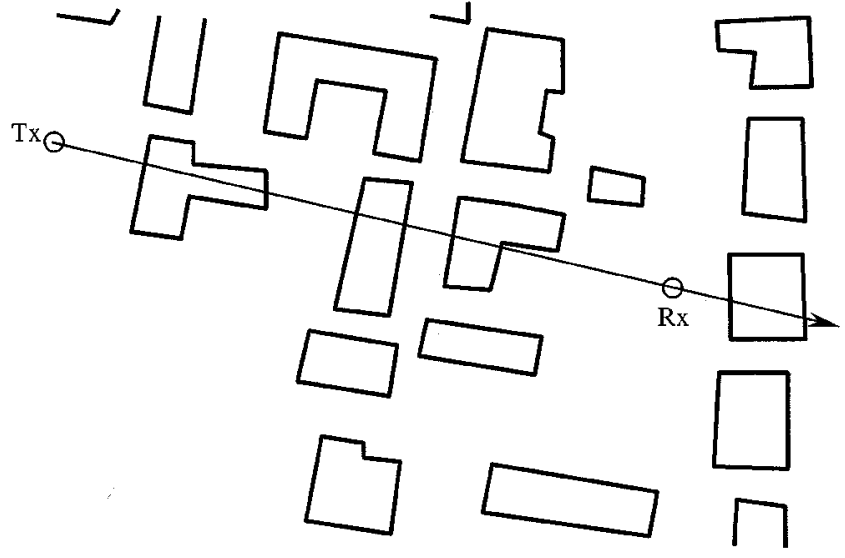

Fig. 8. Determination of over-rooftop rays.

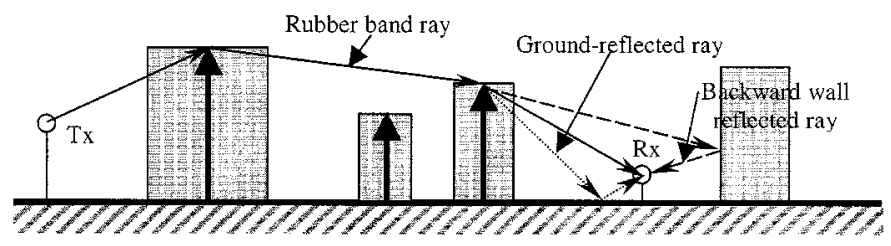

Fig. 9. Vertical profile used to determine the over rooftop rays. Buildings can be approximated by knife edges. Only main rays are shown and taken into account.

ground-reflected ray is the one traveling from the transmitter to the image of the receiver with respect to the ground, which is coincident with the receiver when projected to the horizontal plane, as shown in Fig. 7. Using the height information of vertical walls, we can also determine if a 2-D ray is a 3-D ray with ground reflection. For example, from the 2-D ray tracing, the unfolded ray lengths $D, D_{1}$ and $D_{2}$ are known (Fig. 7(c)). Then the heights of $p_{1}$ and $p_{2}$ can be calculated by

$$
\begin{aligned}
& h_{1}=H_{r}+\left(H_{t}-H_{r}\right) \frac{D_{1}}{D} \\
& h_{2}=-H_{r}+\left(H_{t}+H_{r}\right) \frac{D_{1}}{D} .
\end{aligned}
$$

If $h_{1}\left(h_{2}\right)$ is greater than $H_{2}$, the height of the wall, the reflected ray is not valid.

The diffracted rays by the vertical edges can be traced in a similar manner.

\section{Over-RoOfTOP RAY TRACING AlgORITHMS}

To calculate the over-rooftop rays, it is necessary to know the building profile cut by a vertical plane containing the transmitter and receiver. This can be done very efficiently by launching a ray from Tx to a receiving point using the 2-D ray-tracing method. Fig. 8 shows a ray launched from Tx, going through $\mathrm{Rx}$, and hitting a series of edges, which determines the vertical building profile. Using the height information of edges, the building profile may then be constructed as shown in Fig. 9. Then the buildings are replaced by knife edges and the main rays are traced. The first ray is the "rubber band" ray that connects Tx and Rx. The second ray is the ground reflected ray, and the third one is the backward wall-reflected ray, as shown in Fig. 9. 


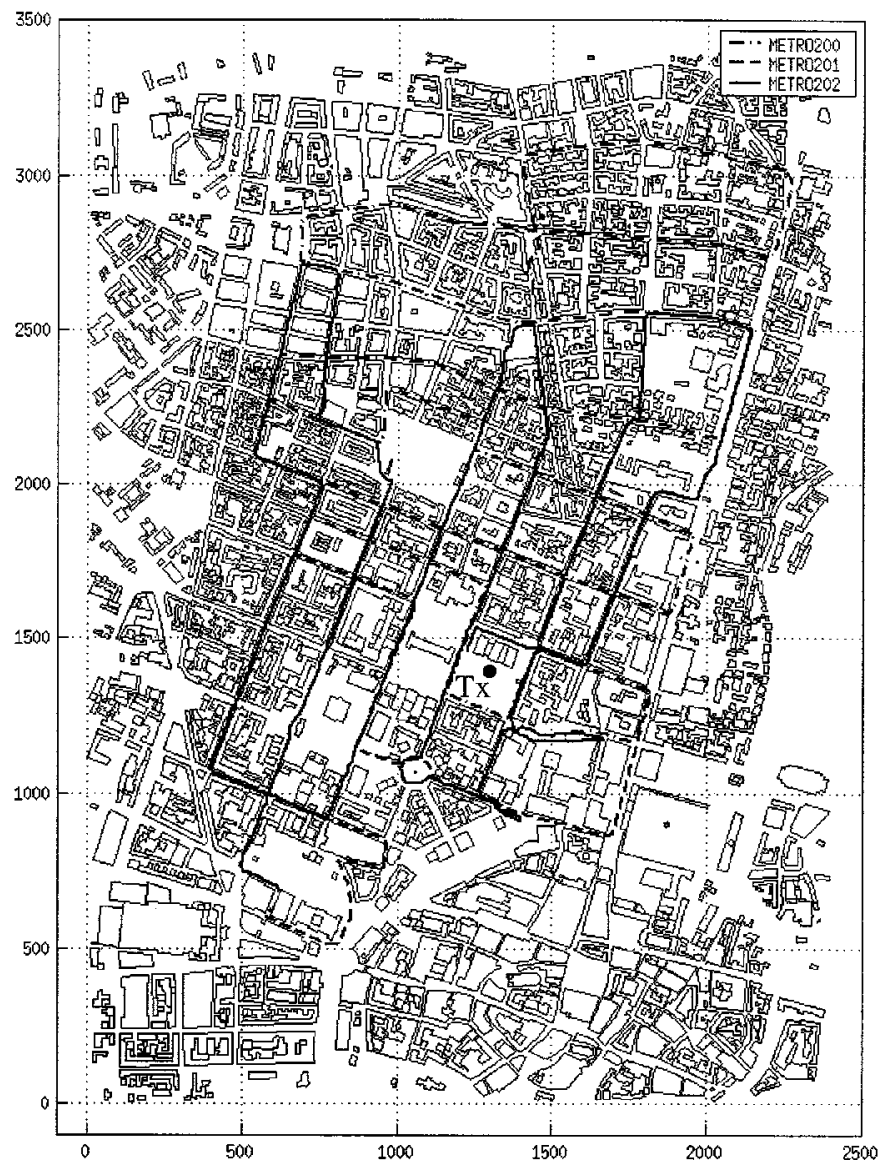

(a)

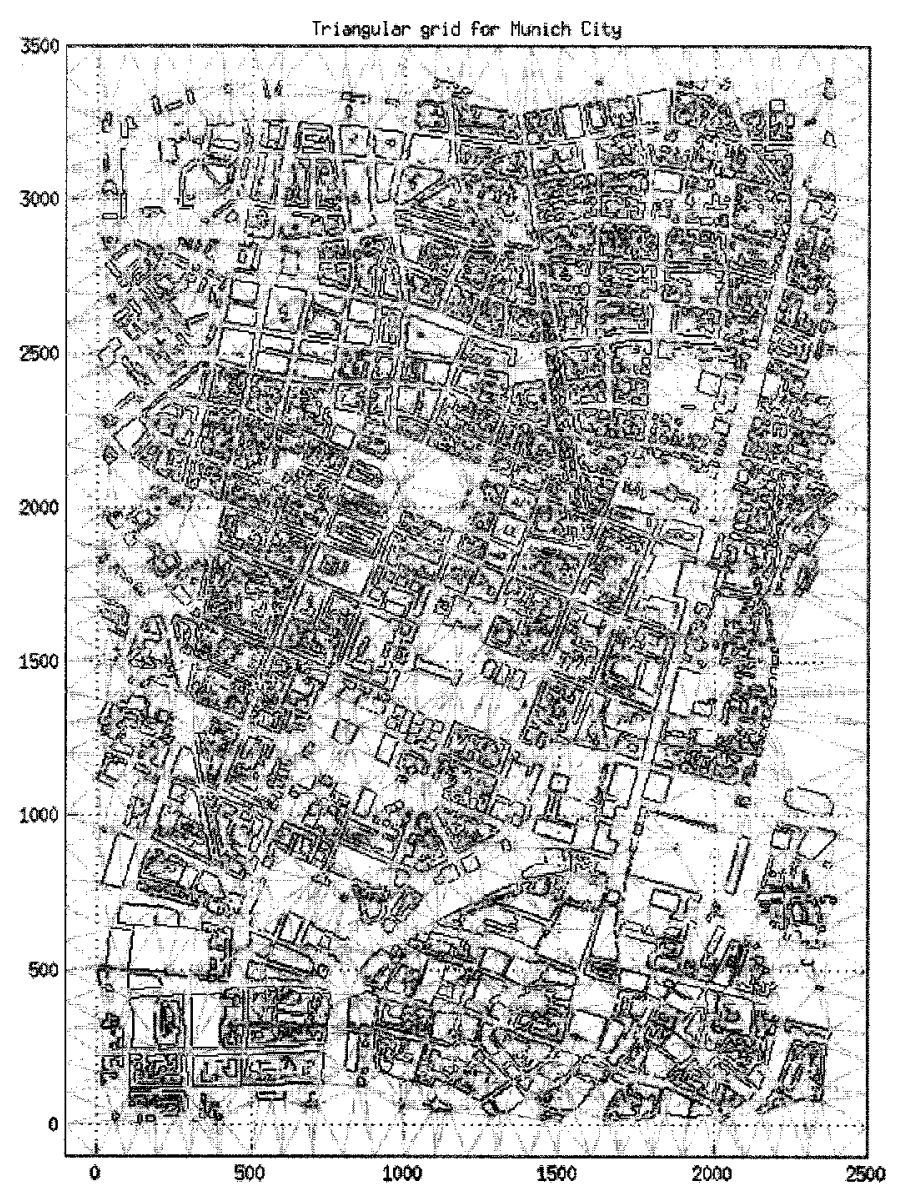

(b)

Fig. 10. (a) Building geometry of Munich City. Also shows three routes (i.e., METRO200, METRO201, and METRO202) used in the experimental measurements and the path losses along these routes were compared with the simulation results using the new ray-tracing method. Note that an outer boundary is added and many dummy edges are inserted in the triangular grid. All dimensions are in meters. b) The triangular grid used in the ray-tracing simulation.

\section{Simulation RESUlTS AND COMPARISON With EXPERIMENTAL DATA}

In an attempt to verify the accuracy and computational efficiency of the proposed method, calculations were made for one of the propagation sites that was comprehensively studied by the European COST 231 working group. Experimental results for Munich City have been amply reported and hence used as the test case for our method.

Fig. 10(a) shows the top view of Munich City and Fig. 10(b) the triangular grid used in our simulation. In this test case, there are 2088 buildings and 17445 walls. The transmitter position and the routes of measurement are also shown Fig. 10(a). These were determined through the measurement efforts established by the COST 231 working group. After triangulation, there are 31156 triangles and 46796 edges. Note that the original building database of Munich is in the form of vectors and was reformatted to fit our use.

Fig. 11 shows the comparison of the simulated and measured path losses for route METRO202. Table I gives a comprehensive comparison of our simulation results with several other results for all three routes (i.e., METRO200, METRO201, and METRO202, as shown in Fig. 10(a)) in Munich City. It can be seen that the proposed method provides results with very good accuracy as compared with others. The details of these models can be found in [9].

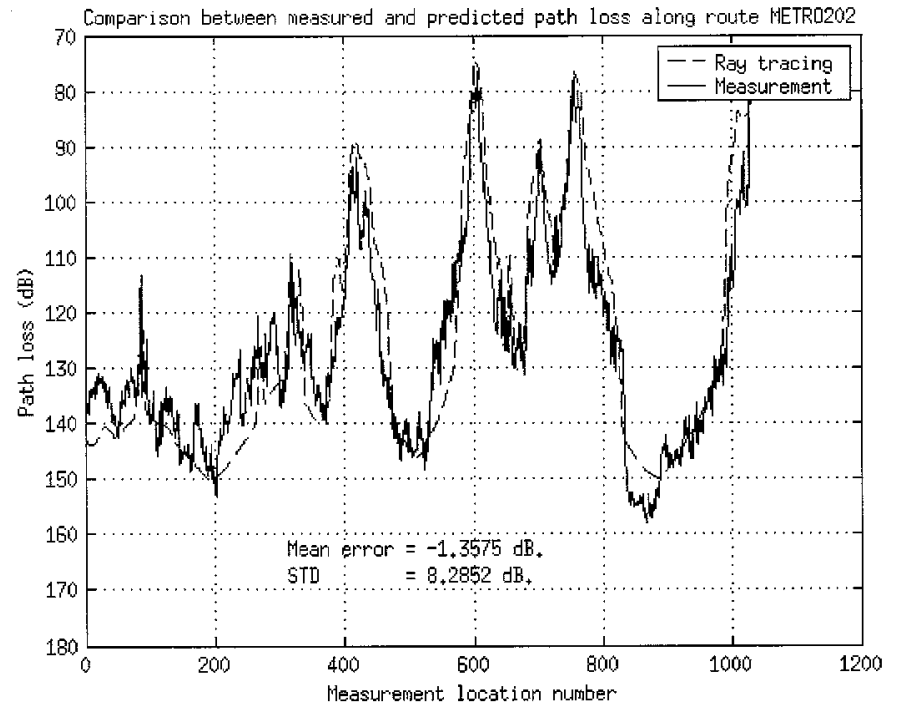

Fig. 11. Comparison with the measured path loss for Munich City.

The total ray-tracing time for all three routes is $3.47 \mathrm{~s}$, running on a Sun Workstation Enterprise $420 \mathrm{R}, 450 \mathrm{MHz}$. To compare the overall computational efficiency of our method, CPU time results were compared with those from a commercial software package WaveSight (http://www.wavecall.com/pre- 
TABLE I

COMPARISON WITH OTHER SIMULATIONS

\begin{tabular}{l|c|c|c|c|c|c|c}
\hline Prediction model & \multicolumn{2}{|c|}{$\begin{array}{c}\text { METRO200 } \\
(970 \text { points })\end{array}$} & \multicolumn{2}{c|}{$\begin{array}{c}\text { METRO201 } \\
(355 \text { points }\end{array}$} & \multicolumn{2}{c|}{$\begin{array}{c}\text { METRO202 } \\
(1031 \text { points })\end{array}$} & Average \\
\hline & $\begin{array}{c}\text { STD } \\
(\mathrm{dB})\end{array}$ & $\begin{array}{c}\text { mean } \\
(\mathrm{dB})\end{array}$ & $\begin{array}{c}\text { STD } \\
(\mathrm{dB})\end{array}$ & $\begin{array}{c}\text { mean } \\
(\mathrm{dB})\end{array}$ & $\begin{array}{c}\text { STD } \\
(\mathrm{dB})\end{array}$ & $\begin{array}{c}\text { mean } \\
(\mathrm{dB})\end{array}$ & $\begin{array}{c}\text { STD } \\
(\mathrm{dB})\end{array}$ \\
\hline Ericsson & 6.7 & 0.3 & 7.1 & 2.3 & 7.5 & 1.4 & 7.1 \\
\hline CNET & 6.9 & -2.1 & 9.5 & -3.6 & 5.6 & -0.2 & 7.3 \\
\hline PTT (RT) & 14.6 & -6.1 & 15.5 & -6.7 & 12.3 & -1.1 & 14.1 \\
\hline PTT (TLM) & 13. & 0.8 & 21.7 & 6.7 & 12.9 & 6.5 & 16.1 \\
\hline COST-WI & 7.7 & 10.8 & 5.9 & 15.4 & 7.3 & 16.3 & 7.0 \\
\hline Uni.-Valencia & 8.7 & 0.2 & 7.0 & -6.6 & 10.3 & -7.4 & 8.7 \\
\hline CSELT & 10.4 & 21.8 & 12.3 & 16.1 & 13.3 & 20.6 & 12.0 \\
\hline PTT (MCOR) & 7.0 & -3.3 & 6.2 & -0.1 & 7.6 & -1.1 & 6.9 \\
\hline Villa Griffone Lab & 6.3 & -1.7 & 10.9 & -6.3 & 6.8 & -5.5 & 8.0 \\
\hline Uni.-Karlsruhe & 8.5 & -4.3 & 9.1 & 2.4 & 8.6 & -1.0 & 8.7 \\
\hline This paper & 7.1 & -2.6 & 6.2 & -0.7 & 8.3 & -1.4 & 7.2 \\
\hline
\end{tabular}

diction.html). For this commercial software, the CPU time is 420 s on a Pentium II 266 for route METRO202, while our CPU time for the same route is $1.5 \mathrm{~s}$. It is understood that calculations were made on different computers, but taking these differences into account (even in an approximate sense) it may be clearly observed that the proposed method provides significant advantages regarding the computational efficiency.

\section{CONCLUSION}

In this paper, a computationally efficient propagation prediction model was presented. The approach is based on 2-D triangulation of the propagation environments and the use of straightforward vector algebra to determine the path of the propagation ray. Basically, the proposed method avoids the use of the usual time-consuming algorithms to determine the appropriate reflecting surfaces in the propagation path. It therefore provides significant advantage in computational efficiency. It is shown that although the triangulation is $2-\mathrm{D}$, it is possible to simulate 3-D environments using the proposed technique. This is accomplished by including additional information on the building heights and considering this information in the determination of true wall hits in the 3-D modeling case.

Accuracy and computational efficiency of the proposed method were evaluated by comparing simulation results with experimental data from the European COST 231 working project and with ray tracing using visibility for a realistic indoor environment. Excellent agreements were observed and considerable savings in CPU time were accomplished. Specifically, an average standard deviation of $7.2 \mathrm{~dB}$ was observed which is considered among the very lowest (ranging from 7.0 to $16.1 \mathrm{~dB}$ ) of currently available techniques that used the same test site. CPU time ratio of the proposed method when compared with the visibility method [32] is about $25 \%$ to $30 \%$. Comparing with one of the available commercial software packages, WaveSight, that was used for the same measurement route in Munich City, the CPU time of the proposed method was $1.5 \mathrm{~s}$ (on a Sun Workstation, Enterprise $420 \mathrm{R}$ ) while the $\mathrm{CPU}$ time of the commercial package was reported to be $420 \mathrm{~s}$ (on a PC, Pentium II 266).

\section{REFERENCES}

[1] J. Walfisch and H. L. Bertoni, "A theoretical model of UHF propagation in urban environments," IEEE Trans. Antennas Propagat., vol. 36, pp. 1788-1796, Dec. 1988.

[2] A. J. Goldsmith and L. J. Greenstein, "A measurement-based model for predicting coverage areas of urban microcells," IEEE J. Select. Areas Commun., vol. 11, pp. 1013-1023, Sept. 1993.

[3] H. H. Xia, H. L. Bertoni, L. R. Maciel, A. Lindsay-Stewart, and R. Rowe, "Radio propagation characteristics for line-of-sight microcellular and personal communications," IEEE Trans. Antennas Propagat., vol. 41, pp. 1439-1447, Oct. 1993.

[4] H. H. Xia, "A simplified analytical model for predicting path loss in urban and suburban environments," IEEE Trans. Veh. Technol., vol. 46, pp. 1040-1046, Nov. 1997.

[5] J. Berg, "A recursive method for street microcell path loss calculations," in Proc. PIMRC'95, 1995, pp. 140-143.

[6] W. C. Y. Lee and D. J. Y. Lee, "Microcell prediction in dense urban area," IEEE Trans. Veh. Technol., vol. 47, pp. 246-253, Feb. 1998.

[7] N. Blaunstein, R. Giladi, and M. Levin, "Characteristics' prediction in urban and suburban environments," IEEE Trans. Veh. Technol., vol. 47, pp. 225-234, Feb. 1998.

[8] D. Har, H. H. Xia, and H. L. Bertoni, "Path-loss prediction model for microcells," IEEE Trans. Veh. Technol., vol. 48, pp. 1453-1462, Sept. 1999.

[9] D. J. Cichon and T. Kurner. Propagation Prediction Models Digital Mobile Radio Toward Future Generation Systems. [Online]. Available: http://www.lx.it.pt/cost231/

[10] S. Y. Seidel and T. S. Rappaport, "Site-specific propagation prediction for wireless in-building personal communication system design," IEEE Trans. Veh. Technol., vol. 43, pp. 879-891, Nov. 1994.

[11] L. Piazzi and H. L. Bertoni, "Achievable accuracy of site-specific path-loss predictions in residential environments," IEEE Trans. Antennas Propagat., vol. 48, pp. 922-930, May 1999.

[12] S. Kim, B. J. Guarino, T. M. Willis III, V. Erceg, S. J. Fortune, R. A. Valenzuela, L. W. Thomas, J. Ling, and J. D. Moore, "Radio propagation measurements and prediction using three-dimensional ray tracing in urban environments at $908 \mathrm{MHz}$ and $1.9 \mathrm{GHz}$," IEEE Trans. Veh. Technol., vol. 48, pp. 931-946, May 1999.

[13] J. E. Dietert, S. Karger, and B. Rembold. Statistical channel modeling based on raytracing simulations. presented at COST259. [Online]. Available: http://www.lx.it.pt/cost259/

[14] J. E. Dietert and B. Rembold. Stochastic channel model for outdoor applications based on raytracing simulations. presented at COST259. [Online]. Available: http://www.lx.it.pt/cost259/

[15] R. P. Torres, S. Loredo, L. Valle, and M. Domingo, "An accurate and efficient method based on ray-tracing for the prediction of local flat-fading statistics in picocell radio channels," IEEE J. Select. Areas Commun., vol. 19, pp. 170-178, Feb. 2001. 
[16] K. Rizk, J. Wagen, and F. Gardiol, "Two-dimensional ray-tracing modeling for propagation in microcellular environments," IEEE Trans. Veh. Technol., vol. 46, pp. 508-517, May 1997.

[17] K. Rizk, J. F. Wagen, and F. Gardiol, "Influence of database accuracy on two-dimensional ray-tracing-based predictions in urban microcells," IEEE Trans. Veh. Technol., vol. 49, pp. 631-642, Mar. 2000.

[18] L. R. Maciel, H. L. Bertoni, and H. H. Xia, "Unified approach to prediction of propagation over buildings for all ranges of base station antenna height," IEEE Trans. Veh. Technol., vol. 42, pp. 41-45, Feb. 1993.

[19] W. Zhang, J. Lahteenmaki, and P. Vainikainen, "A practical aspect of over-rooftop multiple-building forward diffraction from a low source," IEEE Trans. Electromagn. Compat., vol. 41, pp. 115-119, May 1999.

[20] J. Li, J. Wagen, and E. Lachat, "Propagation over rooftop and in the horizontal plane for small and micro-cell coverage predictions," Proc. IEEE 47th VTC'97, vol. 2, pp. 1123-1127, 1997.

[21] D. Erricolo and P. L. E. Uslenghi, "Two-dimensional simulator for propagation in urban environments," IEEE Trans. Veh. Technol., vol. 50, pp. 1158-1168, July 2001.

[22] G. Liang and H. L. Bertoni, "Review of ray modeling techniques for site specific propagation prediction," in Wireless Communications: TDMA Versus CDMA, S. G. Glisic and P. A. Leppanen, Eds. Norwell, MA: Kluwer, 1997, pp. 323-343.

[23] — "A new approach to 3-D ray tracing for propagation prediction in cities," IEEE Trans. Antennas Propagat., vol. 46, pp. 853-863, June 1998.

[24] S. Jan and S. Jeng, "A novel propagation modeling for microcellular communications in urban environments," IEEE Trans. Veh. Technol., vol. 46, pp. 1021-1026, Nov. 1997.

[25] H. Zhu, J. Takada, K. Araki, and T. Kobayashi, "Verification of a two-dimensional/three-dimensional hybrid ray-tracing method for spatiotemporal channel modeling," Radio Sci., vol. 36, no. 1, pp. $53-66,2001$.

[26] G. E. Athanasiadou and A. R. Nix, "Investigation into the sensitivity of the power predictions of a microcellular ray tracing propagation model," IEEE Trans. Veh. Technol., vol. 49, pp. 1140-1151, July 2000.

[27] V. Erceg, S. J. Fortune, J. Ling, A. J. Rustako Jr., and R. A. Valenzuela, "Comparisons of a computer-based propagation prediction tool with experimental data collected in urban microcellular environments," IEEE J. Select. Areas Commun., vol. 15, pp. 677-684, May 1997.

[28] K. Rizk, R. Valenzuela, S. Fortune, D. Chizhik, and F. Gardiol, "Lateral, full-3-D and vertical plane propagation in microcells and small cells," Proc. IEEE 47th VTC'98, vol. 2, pp. 998-1002, 1998.

[29] M. Barbiroli, C. Carciofi, G. Falciasecca, M. Frullone, and P. Grazioso, "A measurement-based methodology for the determination of validity domains of prediction models in urban environment," IEEE Trans. Veh. Technol., vol. 49, pp. 1508-1515, Sept. 2000.

[30] G. E. Athanasiadou and A. R. Nix, "A novel 3-D indoor ray-tracing propagation model: The path generator and evaluation of narrow-band and wide-band predictions," IEEE Trans. Veh. Technol., vol. 49, pp. $1152-1168$, July 2000.

[31] M. F. Catedra, J. Perez, F. S. de Anana, and O. Gutierrez, "Efficient ray-tracing techniques for three-dimensional analyzes of propagation in mobile communications: Application to picocell and microcell scenarios," IEEE Antennas Propagat. Mag., vol. 40, pp. 15-28, Apr. 1998.

[32] F. A. Agelet, A. Formella, J. M. H. Rabanos, F. I. de Vicente, and F. P Fontan, "Efficient ray-tracing acceleration techniques for radio propagation modeling," IEEE Trans. Veh. Technol., vol. 49, pp. 2089-2104, Nov. 2000.

[33] J. G. Cleary and G. Wyvill, "Analysis of an algorithm for fast ray tracing using uniform space division," The Visual Comput., no. 4, pp. 65-83, 1988.

[34] Z. Yun, M. F. Iskander, and Z. Zhang, "Fast ray tracing procedure using space division with uniform rectangular grid," Electron. Lett., vol. 36, no. 10, pp. 895-897, May 2000.

[35] Z. Zhang, Z. Yun, and M. F. Iskander, "Ray tracing method for propagation models in wireless communication systems," Electron. Lett., vol. 36, no. 5, pp. 464-465, Mar. 2000.

[36] S. F. Fortune, D. M. Gay, B. W. Kernighan, O. Landron, R. A. Valenzuela, and M. H. Wright, "WISE design of indoor wireless systems: Practical computation and optimization," IEEE Comput. Sci. Eng., pp. $58-68,1995$.

[37] J. R. Shewchuk. (1996) A two-dimensional quality mesh generator and Delaunay triangulator. [Online]. Available: http:// www.cs.cmu.edu/quake/triangle.html

[38] J. O'Rourke, Computational Geometry in C, Cambridge, U.K.: Cambridge Univ. Press, 1995

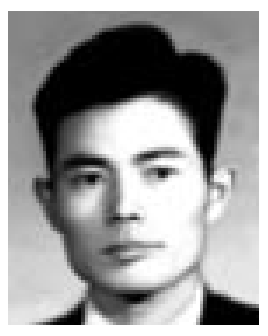

Zhengqing Yun (M'98) received the Ph.D. degree in electrical engineering from Chongqing University, China, in 1994.

From 1995 to 1997, he was a Postdoctoral Fellow in the State Key Laboratory of Millimeter Waves, Southeast University, Nanjing, China. Since 1997, he has been a Research Assistant Professor in the Electrical Engineering Department, University of Utah, Salt Lake City. He is now The Hawail Center for Advanced Communication, College of Engineering, University of Hawaii at Manoa, Honolulu. His research interests include development of numerical methods, modeling of radio propagation for wireless communications systems, and design and simulation of antennas.

Dr. Yun was the recipient of the 1997 Science and Technology Progress Award (1st Class) presented by The State Education Commission of China.

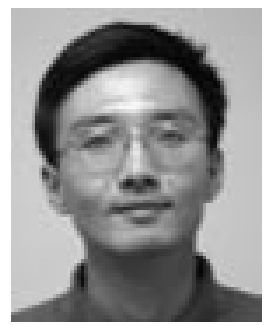

Zhijun Zhang (M'00) received the B.S. and M.S. degrees in electrical engineering from University of Electronic Science and Technology of China, Chengdu, China, and the Ph.D. degree in electrical engineering from Tsinghua University, Beijing, China, in 1992, 1995 and 1999, respectively.

From 1999 to 2001, he was a Postdoctoral Fellow in the Department of Electrical Engineering, University of Utah, Salt Lake City. In 2001, he was appointed Research Assistant Professor in same department. He is now with The Hawaii Center for Advanced Communications, College of Engineering, University of Hawaii at Hanoa, Honlulu. His research interests include the areas of RF circuit design, antenna design and wireless communication multipath environment simulation.

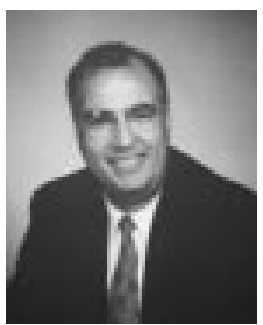

Magdy F. Iskander (S'72-M'76-SM'84-F'93) is the director of the Hawaii Center for Advanced Communications, College of Engineering, University of Hawaii at Manoa, Honolulu. He was a Professor of Electrical Engineering and the Director of the Center of Excellence for Multimedia Education and Technology (formerly the NSF/IEEE Center for Electromagnetics Education) at the University of Utah, Salt Lake City. From 1998 to 1999, he was appointed Program Director of the Physical Foundation of Enabling Technologies, in the Electrical and Communication Systems, Division of the National Science Foundation (NSF). At NSF he formulated and directed a "Wireless Information Technology and Networks" initiative in the Engineering Directorate. This wireless communications initiative resulted in funding over 29 projects in the microwave/millimeter wave devices, RF micromachining and MEMS, propagation, and the antennas areas. In 1986, Dr. Iskander established the Engineering Clinic Program to attract industrial support for projects to be performed by undergraduate engineering students at the University of Utah. Since then, more than 110 projects have been sponsored by 33 corporations from across the United States. The Clinic Program now has an Endowed Chair for the Director. $\mathrm{He}$ is author of the textbook Electromagnetic Fields and Waves (Englewood Cliffs, NJ: Prentice-Hall, 1992), and a revised version published by Waveland press, Inc., in 2000. He is editor to the CAEME Software Books, Vol. I,and Vol. II, in 1991 and 1994, respectively. and edited four other books Microwave Processing of Materials, (Pittsburgh, PA: Materials Research Soc.), in 1990, 1992, 1994, and 1996, respectively. He was the editor of two special issues for the Journal of Microwave Power, in March 1983 and September 1983; a special issue of the ACES Journal on Computer-Aided Electromagnetics Education and the Proceedings of the International Conference on Simulation and Multimedia in Engineering Education, 1995 and 1996. He is presently coeditor of the IEEE TRANSACTIONS ON ANTENNAS AND PROPAGATION Special Issue on Wireless Communications. In addition, he has published over 170 papers in technical journals, has 8 patents, and made numerous presentations in technical conferences. He is the founding editor of Computer Applications in Engineering Education (CAE), (New York: Wiley) published from 1993 to the present. His research interests include computational electromagnetics, antenna design and propagation models for wireless communications, microwave processing of materials, biological effects of EM radiation, and the development of multimedia applications. 
Dr. Iskander received the Curtis W. McGraw ASEE National Research Award for outstanding early achievements, the ASEE George Westinghouse National Award for innovation in engineering education, and the 1992 Richard R. Stoddard Award from the IEEE Electromagnetic Compatability Society. In 2000, he received the University of Utah Distinguished Teaching Award and received the excellence in publishing award in 1993 for Computer Applications in Engineering Education (CAE) in the category of Technology, Science, and Medicine. He was the General Chairman of the 1996 Frontiers in Education Conference sponsored by the Computer and Education Societies of IEEE and the General Chair for the 2000 IEEE APS International Symposium and URSI meeting. He was a member of the IEEE APS AdCom from 1997 to 1999 and is presently the Vice President of the Antennas and Propagation Society of IEEE. He was a member of the WTEC panel on wireless technology and Chair of the Asia Telecommunication panel sponsored by the Department of Defense (DoD) and organized by the International Technology Research Institute (ITRI). He is a member of the National Research Council Committee on Microwave Processing of Materials. He was a Distinguished Lecturer for the Antennas and Propagation Society of IEEE from 1994 to 1997, and gave lectures in Brazil, France, Spain, China, Japan, and a number of U.S. Universities and IEEE chapters. 